\title{
ASR-FANET: An adaptive SDN-based routing framework for FANET
}

\author{
Alaa Taima Albu-Salih', Hayder Ayad Khudhair ${ }^{2}$ \\ ${ }^{1}$ Ministry of Education, General Directorate for Education in Al-Qadisiyah, Iraq \\ ${ }^{2}$ Ministry of Education, General Directorate for Education in Al-Najaf Al-Ashraf, Iraq
}

\section{Article Info \\ Article history: \\ Received Sep 14, 2020 \\ Revised Mar 20, 2021 \\ Accepted Apr 5, 2021}

\section{Keywords:}

FANET

IoT

OLSR

SDN

UAV

\begin{abstract}
Flying ad hoc network (FANET) is widely used in many military, commercial and civilian applications. Compared with mobile adhoc network (MANET) and vehicular ad hoc network (VANET), FANET holds unique characteristics such as high mobility, intermittent links and frequent topology changes, which cause a challenging task in the design of routing protocols. A novel adaptive software defined networking (SDN)-based routing framework for FANET called ASR-FANET is proposed in this article to solve the above challenges. The ASR-FANET framework is mainly composed of three important parts, which are the topology discovery mechanism, statistics gathering mechanism and route computation mechanism. In topology discovery mechanism, the periodic information about network topology is collected, including nodes and links. In statistics gathering mechanism, the status of the wireless network connection and flight statistics are collected. In route computation mechanism, the optimal path is calculated based on link costs. The performance of ASR-FANET framework is also has been evaluated by comprehensive simulations. The simulation results show that proposed framework is much better than other traditional protocols in packet delivery fraction, average end to end delay, normalized routing load, packet loss and throughput.
\end{abstract}

This is an open access article under the CC BY-SA license.

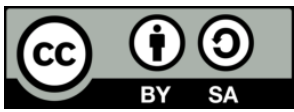

Corresponding Author:

Alaa Taima Albu-Salih

Department of Computer Science

Al Quadisiya University

58002 University Road, Aldiwanyia, Al Quadisiya, Iraq

Email: alaa.taima@qu.edu.iq

\section{INTRODUCTION}

Unmanned aerial vehicles (UAVs), also known as drones, played a significant role in different civil and military applications such as reconnaissance, traffic monitoring, battlefield surveillance, border surveillance, forestry fire monitoring, animal tracking, humanitarian observations [1]. Instead of using one large UAV, the use of a swarm of small UAVs reveals many advantages in terms of efficiency, adaptability, flexibility and easy deployment [2]. The use of multiple UAVs, has helped to create the foundation of a new kind of network called flying ad hoc network (FANET) [3], [4].

FANET can be considered a special sub-class of mobile adhoc network (MANET) in which the connecting nodes are UAVs [5]. Therefore, a single UAV cannot be regarded as a FANET and is only valid for multiple UAVs. All multiple UAV networks cannot be regarded as a FANET. The communication between UAVs should be in ad hoc manner to create FANET [6]. Additionally, FANET can also be classified as a subgroup of vehicular ad hoc network (VANET), which is also a sub-class of MANET. Figure 1 shows the relationship between FANET, VANET, and MANET [3], [7]. 


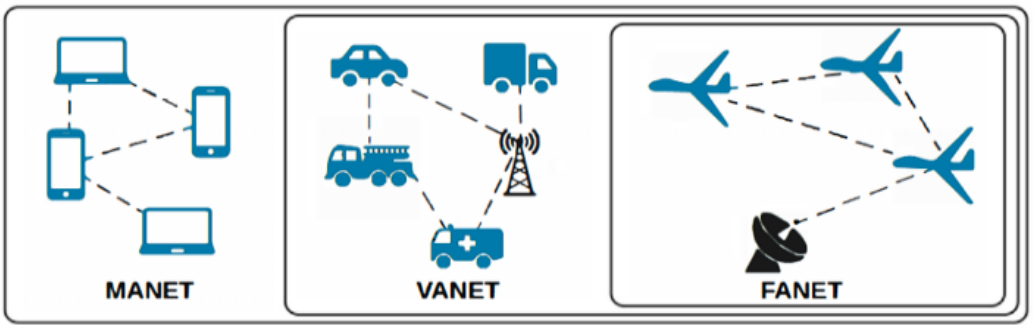

Figure 1. MANET, VANET and FANET networks

The most certain differences between the FANETs and other adhoc networks (MANET and VANET), is the dynamic topology of network, the speed of nodes and the mobility of the nodes. Additionally, FANET differs from MANET and VANET in connection, QoS, node movement features, and data delivery. The comparison between MANET, VANET and FANET with different criteria are given in Table 1 [8], [9].

FANET nodes (UAV) are used to collect data from the internet of things (IoT) as mobile collectors [10]. Indeed, each single UAV in FANET can play a major role on the IoTs, which consists of devices with a small battery capacity sensor [11]. These devices can not usually be distributed over long distances, due to the energy limitation of IoT devices. Thus, UAV will travel dynamically towards IoT devices, gather data and transfer it to other UAVs in FANET network that are outside the communication ranges of IoT devices [12], as seen in Figure 2.

Table 1. Differences between FANET, VANET and MANET [3]

\begin{tabular}{|c|c|c|c|}
\hline Criteria & MANET & VANET & FANET \\
\hline Node mobility & Low & High & Very High \\
\hline Mobility model & Arbitrary & Steady & $\begin{array}{l}\text { Usually predetermined, but special mobility } \\
\text { models for independent multi-UAV systems }\end{array}$ \\
\hline Topology change & Slow & Average & Fast \\
\hline $\begin{array}{l}\text { Radio propagation } \\
\text { model }\end{array}$ & $\begin{array}{l}\text { Very close to ground, LoS is } \\
\text { not accessible for all cases }\end{array}$ & $\begin{array}{l}\text { Close to ground, LoS is not } \\
\text { accessible for all cases }\end{array}$ & $\begin{array}{l}\text { High above the ground level, Line of Sight } \\
\text { is accessible for most of the cases }\end{array}$ \\
\hline Computational power & Limited & Average & Very BIG \\
\hline Localization & GPS & GPS, AGPS, DGPS & GPS, AGPS, DGPS, IMU \\
\hline
\end{tabular}

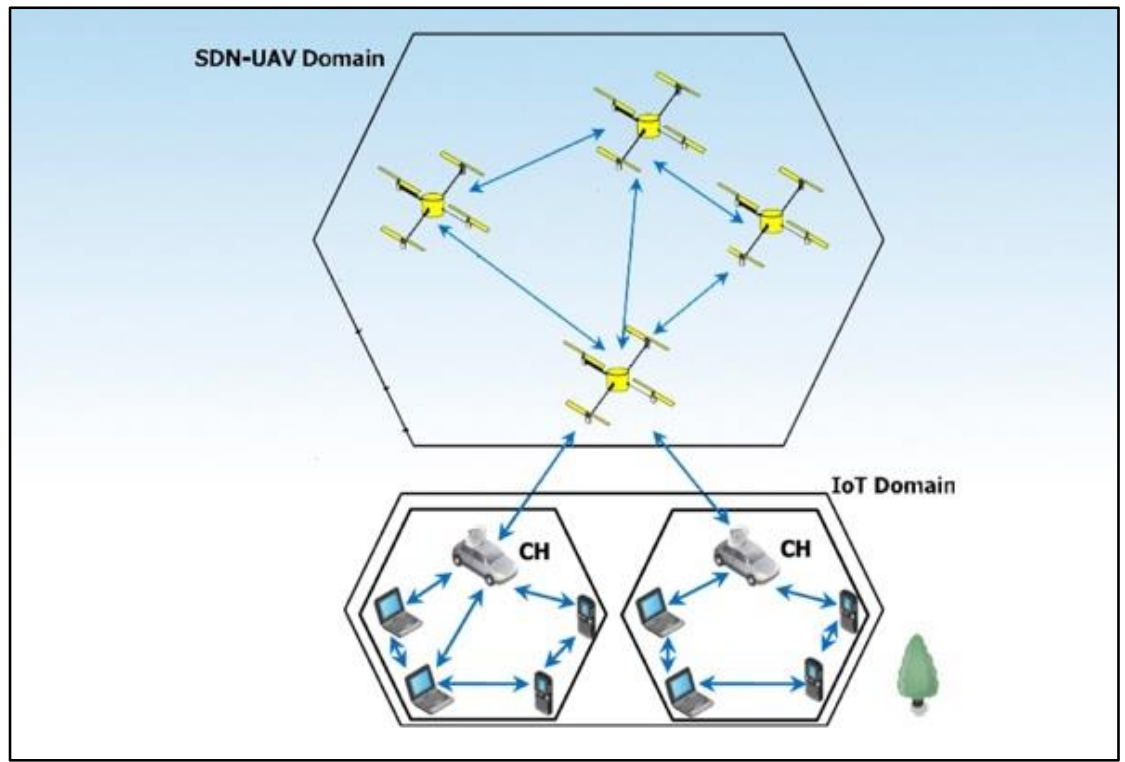

Figure 2. UAV-IoT network

Int J Elec \& Comp Eng, Vol. 11, No. 5, October 2021: 4403 - 4412 
Routing is one of the most challenging issues for FANET because of the specific features of FANET such as fast network topology variance, high mobility and intermittent links between UAV nodes [13]. Current MANET and VANET routing protocols cannot meet all of the FANET requirements network because FANET topology will change more often than standard MANET and VANET topology [14]. As a result, those protocols fail to follow the evolution of the topology of the FANET network. So, there is a big challenge in front of researchers to provide an efficient and reliable routing strategy. Therefore, there is a need of developing new routing strategy for to providing a reliable communication between FANET nodes.

A promising alternative to conventional internet protocol (IP) networks is the software-defined networks (SDN). SDN implements network programmability by separating network control and data plane [15]. The control plane a logically centralized network controller dictates overall network activities, while in the data plane is responsible for transmitting data flows. The SDN architecture enhances the routing strategy 's efficiency based on the information gathered by the SDN controller [16].

The FANET network is characterized for its quick changing network topology, high mobility, intermittent links, energy constraints, and link quality changes. Traditional routing strategies are not able to meet the above FANET requirements. So, we propose a novel adaptive SDN routing framework for FANET (namely, ASR-FANET) considering the dynamic topology of FANET. To optimize the routing and quality of service (QoS) requirements, FANET topology can be dynamically modified using an ASR-FANET framework. The goal of this framework is to enhance the performance of its network while taking into account all the routing constraints, such as fast changing network topology, high mobility, intermittent links and link quality changes. The article provides the contributions: By defining key routing problems in the FANET, we perform an extensive FANET routing investigation which is the main features of this article. The contributions of this paper can be defined is being as:

a. By defining the key challenges of the routing problem in the FANET, we perform an accurate investigation of SDN-based routing in FANET.

b. An Adaptive SDN-based routing framework is proposed for dynamic routing using multiple UAVs in the IoT network. We refer to this framework as (ASR-FANET). This framework includes an ASR-FANET controller that collects the global information of FANET nodes (e.g., speed, location information and residual energy). This controller makes routing decisions and decides how the UAV nodes should move.

c. The performance of this ASR-FANET is compared with two common traditional routing protocols in terms of packet delivery fraction, end2end delay, normalized routing load, packet loss and throughput.

d. Simulation results show that the proposed framework can achieve lower end2end delay, normalizing routing load, and higher throughput and packet delivery fraction.

The rest of this paper is structured is being as. The routing methods in FANET networks are discussed in section 2. Section 3 introduces the suggested framework steps. In section 4, the steps of the suggested framework are presented. Finally, section 5 provides conclusions and recommendations for future study.

\section{RELATED WORKS}

In spite of practical applications of FANET in IoT networks, many technical issues such as routing should be studied. Although traditional routing protocols are designed MANET, they are not necessarily suited to FANET because FANET topology will change more often than standard MANET. A number of routing protocols with different techniques are proposed for FANET trying to increase the packet delivery ratio, reduce the end to end delay, to decrease packet drop, and to be adapted in different situations. Therefore, we first review the relevant SDN-based routing protocols in FANET according to the previous issue, and then examine each of these works and describe the advantages and drawbacks of each work.

The authors Ramaprasath in [17] proposed a novel UAVs communication scheme based SDN. An SDN controller is used to calculate the routes and to monitor information with regards to the network. The main goal of this scheme is to decrease the latency, and increase the throughput Silva et al. [18] exploited the SDN to maintain the topology of FANET. The primary purpose of this protocol is to define and modify the topology so as to maintain the connection between the independent nodes via the relay nodes.

Secinti et al. [19] proposed resilient multi-path routing framework by SDN for dynamic UAV networks. The SDN controller is composed of 4 separate modules: Data acquisition module, transmission time calculation module, end2end resilient multipath routing module, and multi-layer graph modeling module. A multi-layer graph modeling module with SDN controller is used to create separate paths to prevent jammed routes.

Iqbal [20], an SDN-based mechanism for FANET with highly dynamic is proposed. The mechanism is used to predict network disruptions (such as link outages) and maximize availability and performance of network. An SDN is used to make the necessary routing changes for minimizing the effect of the predicted link changes. Z. Yuan [21], novel architecture for mobile sensor networking is proposed for a micro UAV 
using SDN. The goal of this architecture is to enhance network performance and increase the use of conventional mobile networks for wireless channel resources.

Qi et al. [22] developed a TDR algorithm with a coordinator and a controller for the SDN cluster. The controller is used to collect the whole abstract cluster network views, schedule the network resources and also guide the data forwarding. TDR aims to ensure the reliability and delay-sensitive capabilities of various applications with the respective QoS.

Chen et al. [23] developed an ATN architecture based on the SDN for airborne tactical network. (SD-ATN's) software defined-arsitektur network goal is to ensure that the SDN control plane collects the data plane monitoring information in real time. MCF-SD-ATN is a communication protocol is designed to collect monitoring information in efficient manner and ensure a QoS of monitoring information.

Alharthi et al. [24] utilizes the SDN in SDN-based UAV architecture to incorporate a wide variety of deployment scenarios for UAVs. The architecture is useful for use in cases such as network coverage, scanning, and sensing, and is capable of working where network connectivity is not available. Small-and large-scale scenarios have been employed to explain this architecture.

Kirichek et al. [25] designed an FUSN architecture based on the SDN for flying sensor network in which UAVs are used as controllers and sensors. FANET nodes are deployed to create connectivity between ground and a flying data collector as switches. Another node will be configured as the SDN controller for updating the routing tables for the other nodes. This architecture aims to establish efficient routing from mobile sensors located on the ground with the help of UAVs. In Table 2, the previously reviewed works for SDN-based routing solutions are compared in terms of some parameters such as deadline time, optimal deployment of UAVs, the energy consumption of FANET nodes, the optimal number of FANET nodes, and the minimum amount of time to collect data from IoT nodes.

Table 1. Comparison of the reviewed works

\begin{tabular}{|c|c|c|c|c|c|c|c|}
\hline Reference & $\begin{array}{c}\text { Single- } \\
\text { Hop/ } \\
\text { multi-hop } \\
\end{array}$ & $\begin{array}{c}\text { Fixed/ } \\
\text { mobile } \\
\text { nodes }\end{array}$ & $\begin{array}{l}\text { Centralized/ } \\
\text { Distributed }\end{array}$ & $\begin{array}{c}\text { Optimal } \\
\text { positioning } \\
\text { of UAV }\end{array}$ & $\begin{array}{c}\text { Energy } \\
\text { Efficiency }\end{array}$ & $\begin{array}{c}\text { Minimum } \\
\text { travelled } \\
\text { time of UAV }\end{array}$ & Deadline \\
\hline Ramaprasath et al. [17] & Multiple & Fixed & Centralized & No & Yes & No & No \\
\hline Silva et al. [18] & Multiple & Mobile & Centralized & Yes & No & No & No \\
\hline Secinti et al. [19] & Multiple & Fixed & Distributed & No & No & No & No \\
\hline Iqbal et al. [20] & Single & Fixed & Centralized & No & No & No & No \\
\hline Yuan et al. [21] & Single & Mobile & Centralized & No & No & No & No \\
\hline Qi et al. [22] & Single & Mobile & Distributed & No & Yes & No & No \\
\hline Chen et al. [23] & Single & Fixed & Centralized & No & No & No & No \\
\hline Alharthi et al. [24] & Single & Mobile & Centralized & No & Yes & No & No \\
\hline Kirichek et al. [25] & Single & Fixed & Centralized & Yes & No & No & No \\
\hline
\end{tabular}

\section{THE PROPOSED FRAMEWORK}

To address the issue of traditional routing in FANET network, a novel adaptive SDN routing framework for FANET was proposed (namely, ASR-FANET). An adaptive-SDN controller is used in ASRFANET, in which network control is divided between SDN control and UAV (data plane). In this case, the FANET nodes have the decisions to carry data with the distributing routing protocols, without the SDN controller involving them. In the presence of network failures and instability, non-SDN (traditional) routing protocols such as optimized link state routing (OLSR) can be used to provide a stable network architecture for exploring network topology and routing.

Part of the nodes in the SDN network in ASR-FANET framework will dynamically move from SDN to a conventional distributed routing protocol (e.g. OLSR) and bypass SDN forwarding rules. In distributed manner, FANET nodes can be selected in a specific area where frequent network changes occur. Naturally, the distributed routing protocol will be compatible with these changes faster than the SDN controller. When the connection to the SDN controller becomes more stable, FANET nodes can be reconfigured by the SDN controller and migrated to the SDN again. By transferring the control to the FANET nodes, the SDN architecture more becomes suitable for the FANET environment and more compatible with network modifications.

SDN network is formed by mounting the Open Flow switches on the UAVs and the control facility on a centralized controller. We considered a centralized network in which the locations of UAVs and IoT devices are known to an SDN controller. The controller is aware of the global network topology. We used a single central controller which monitors the overall traffic network.

Since many of the IoT network applications have been spread equally on the network, we also presume that the distribution of IoT nodes in the network would be uniform. Nodes are of two types: Cluster 
head $(\mathrm{CH})$ and cluster member $(\mathrm{CM})$. The $\mathrm{CHs}$ and $\mathrm{CMs}$ are randomly deployed in the network field. Each device would usually has a line of sight view with a given probability towards a particular UAV. This line of sight possibility depends on the environment, the position of the IoT device, the UAV and the elevation angle between UAV and IoT device. In this article, each of the IoT nodes is supposed to be the ability to set their own rates as well as the radius of transmission. All nodes of IoT network are aware of their geographical place based on global positioning system (GPS), and their locations are known to FANET and are used to find optimal route for FANET nodes.

We assume that the of UAV nodes in the FANET will be distributed in adhoc formation. In this article, every UAV has the ability to move at fixed speed and fixed height. UAV with high processing power and memory will be assumed. In this article, every UAV has the ability to move with no obstacles. Every UAV has the ability to move without risk of collision. Based on the above assumptions of FANET and IoT networks, the ASR-FANET framework for designing a novel dynamic routing framework for FANET using the Adaptive SDN can be consisted of three main mechanisms: topology discovery mechanism, statistics gathering mechanism and, and route computation mechanism. As shown in Figure 3, all of these three mechanisms are located in the SDN controller. Below we will explain each of them in detail:

- Topology discovery mechanism

In this mechanism, the periodic information about network topology (nodes and links) is collected. The connectivity information about network is constantly available to other mechanisms in the SDN controller.

- Statistics gathering mechanism

This mechanism collects the status of the FANET flight statistics (speed, GPS data, IMU data) and network connection (delay, RSSI, bit error rate). The information for this mechanism is sent as a reference to create a new flow path.

- Route computation mechanism

This mechanism is used to determine the optimal paths based on data obtained from other mechanisms.

Any UAV in FANET has both LTE and Wi-Fi connectivity. The control connection between UAV and SDN is enabled through LTE connectivity, while the Wi-Fi connectivity provides data communication between the UAVs and IoT devices. The OLSR routing protocol is used for data traffic for the lack of access to the SDN controller. The OLSR routing protocol provides an appropriate response to change the topology events, such as adding/removing UAV nodes and wireless links among them.

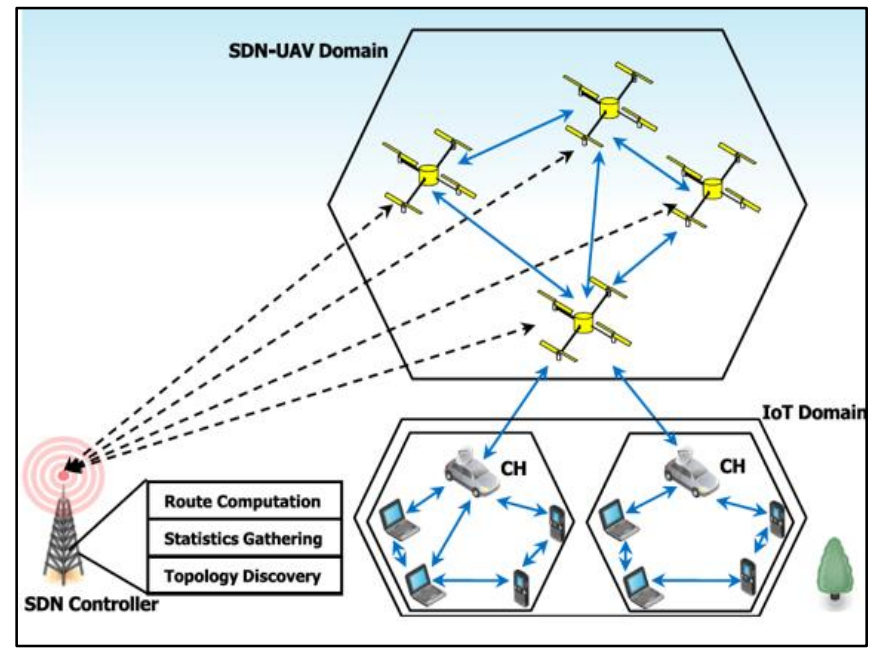

Figure 3. Illustration of ASR-FANET architecture

\section{PERFORMANCE EVALUATION}

To demonstrate the performance of the ASR-FANET framework presented above, we chose one reactive routing protocol (AODV) [26] and one proactive routing protocols (OLSR) [27]. These protocols can be used to evaluate the performance evaluation of the suggested framework for dynamic routing for FANET network. The following metrics are used to evaluate the effectiveness of ASR-FANET framework: - Throughput 
- Package delivery ratio (PDF)

- Packet loss (PL)

- Normalized routing load (NRL)

- End to end delay (E2E Delay)

\subsection{Simulation setup}

In our simulation, the size of the $800 \times 800 \mathrm{~m} 2$ network is assumed. The simulation was performed using python software as a platform for implementation on a system with a processor $2.3 \mathrm{GHz}$ Quad-Core Intel Core i5 and 8 gigabytes of main memory. The rest of simulation parameters are enlightened in Table 2. To implement the research, we developed the previous proposed ASR-FANET approach with POX controller and we used OpenFlow protocol as the southbound to collect network statistical information from our network. A routing mechanism which got the statistical information to make a decision for data plane.

Table 2. Simulation parameters

\begin{tabular}{cc}
\hline Parameter & Value \\
\hline SDN Controller & POX \\
MAC Type & 802.11 \\
Radio Wave Propagation & TwoRayGround \\
Antenna & Omni Antenna \\
Simulation Time & 200 seconds \\
Mobility Model & Random Walk \\
Area size & $800 \times 800 \mathrm{~m} 2$ \\
No. of IoT Nodes & 200 \\
No. of UAV & 5101520 \\
UAV's Speed & $10203040 \mathrm{~m} / \mathrm{s}$ \\
UAV's Heights & $70 \mathrm{~m}$ \\
Transmission Range & $40 \mathrm{~m}$ \\
UAV Elevation Angles & $45 \mathrm{deg}$ \\
Transmission Bit Rate & $200 \mathrm{kbps}$ \\
Packet Size & $2000 \mathrm{bit}$ \\
\hline
\end{tabular}

\subsection{Results and analysis}

In this section, some comparisons are made between ASR-FANET and several algorithms, which include AODV algorithm and OLSR algorithm in terms of PDR, E2E delay, packet loss, throughput, and NRL. ASR-FANET's performance is tested using two scenarios.

a. Scenario 1: Different number of UAV nodes

In this scenario, the effect of the number of UAV nodes on the effectiveness of ASR-FANET of is investigated. Figure 4. shows the performance metrics of proposed ASR-FANET and two adhoc routing protocols for scenario 1. According to Figure 4(a), the PDF of ASR-FANET is the best due to the proposed ASR-FANET calculates paths based on the link utilizations. PDF in AODV and OLSR performed the worst. Figure 4(b) displays that ASR-FANET also outperformed AODV and OLSR when evaluated using the packet losses metric. The packet losses are decreased when the number of nodes increased. Figure 4(c) shows the AED is decreased when the number of nodes increased. AED in our proposed method is least and in [14] and [15] is highest. In in Figure 4(d), the throughput of ASR-FANET method shows a lower NRL over number of UAVs. This is because the SDN controller can adjust routing paths dynamically to avoid overloaded links. The NRL of these methods is decreased when the number of UAVs increased. In Figure 4(e), the throughput is more significant with ASR-FANET and the throughput is less significant with routing methods in AODV and OLSR because the ASR-FANET finds the optimal paths adaptively regarding the condition of network.

b. Scenario 2: Various Speed of UAV

This scenario considers the various speed of UAV to demonstrate the efficiency of ASR-FANET. Figure 5 shows the performance metrics of proposed framework and two routing method (AODV and OLSR) for scenario 2. According to Figure 5(a) the PDF were decreased when the UAV speed was increased due to the proposed ASR-FANET calculates paths based on the link utilizations. The proposed method has high PDF while AODV and OLSR are lower. Figure 5(b) shows the number of packets lost in [14] and [15] is highest, while in our proposed method is lowest. ASR-FANET considers adaptive method to reduce the number of dropped packets. The lost packet is increased when the node speed increased. According to Figure 5(c), adaptive routing strategy of the ASR-FANET leads to significant reduction in average E2E. This Figure shows the average E2E increases when the UAVs speed increased. In Figure 5(d), the NRL of these protocols is increases with high speed for all methods. The method-based Adaptive SDN has low NRL than 
other routing methods while the methods in AODV and OLSR have high NRL. When the UAVs' speed increases, NRL increases significantly, due to the numbers of link changes is increased. In Figure 5(e), the throughput of our proposed method was decreased when the node speed was increased. The proposed method has high throughput and PDF while the methods in traditional routing protocol are lower. This is because ASR-FANET increase the network throughput due to its adaptive approach, making the communication process more efficient.

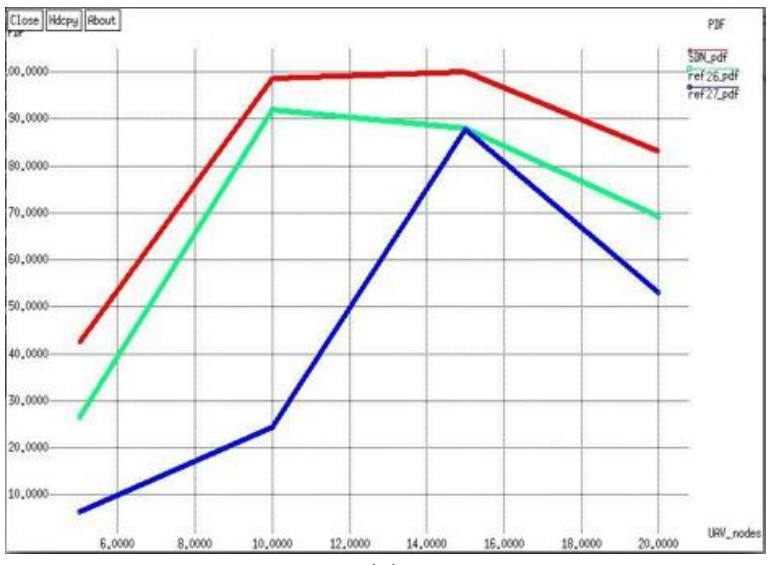

(a)

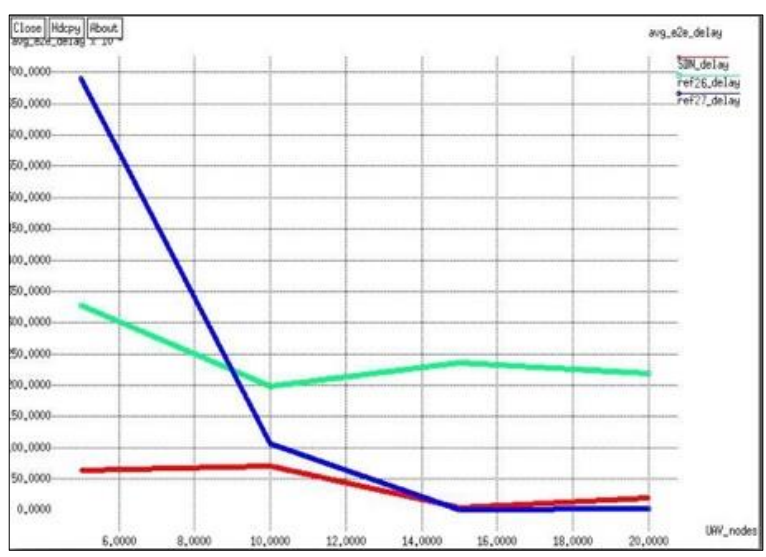

(c)

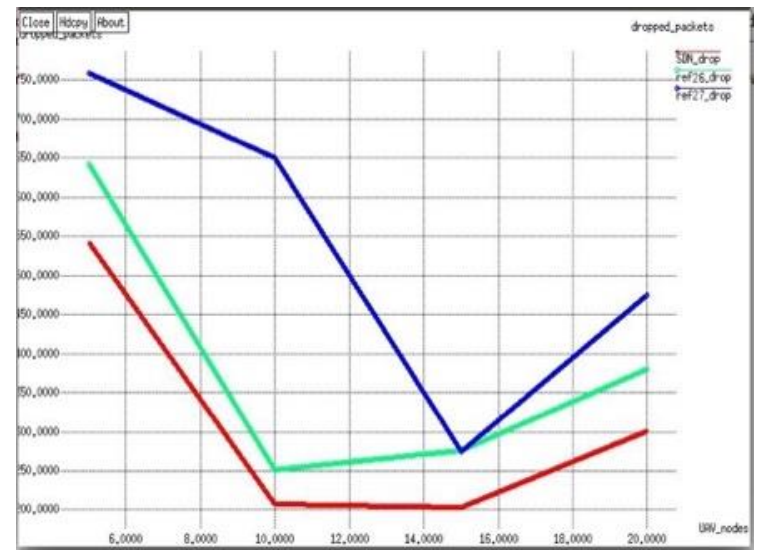

(b)

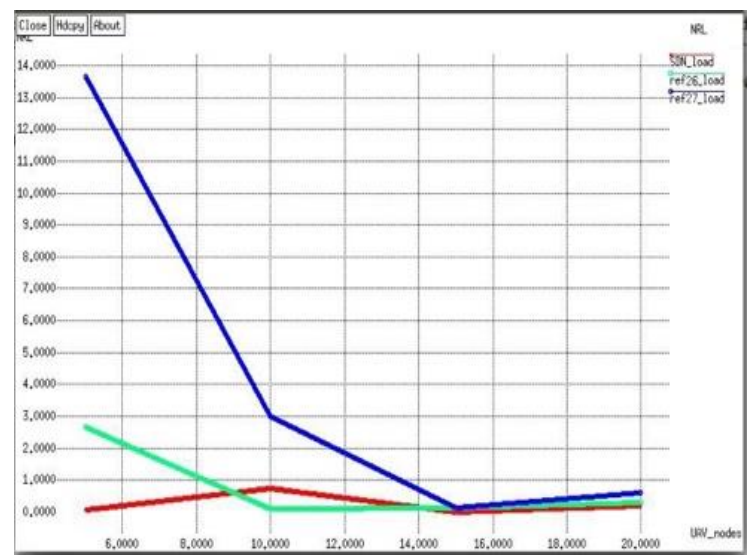

(d)

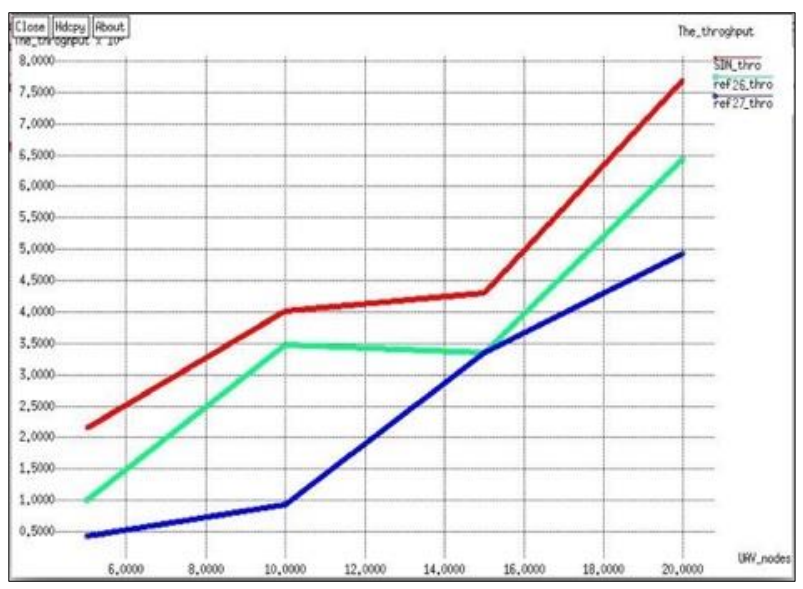

(e)

Figure 4. The performance metrics of ASR-FANET, ref (26) and ref (27) for scenario 1; (a) PDF, (b) dropped packet, (c) E2E delay, (d) NRL, (e) throughput 


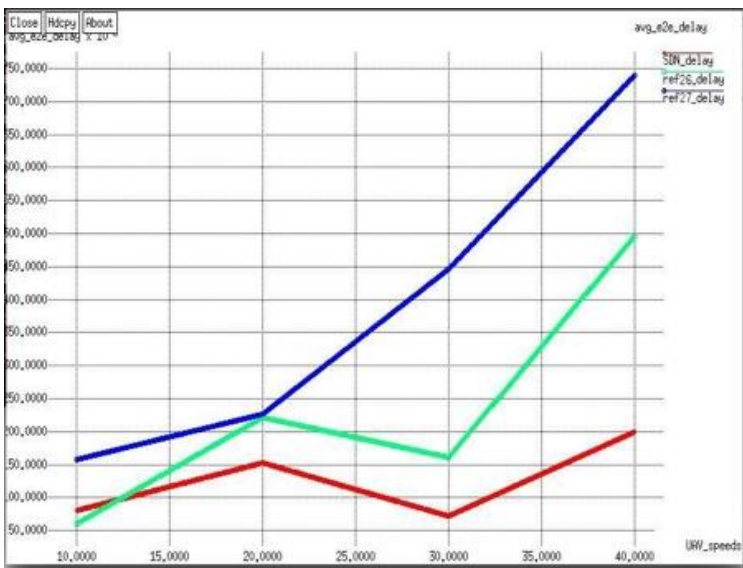

(a)

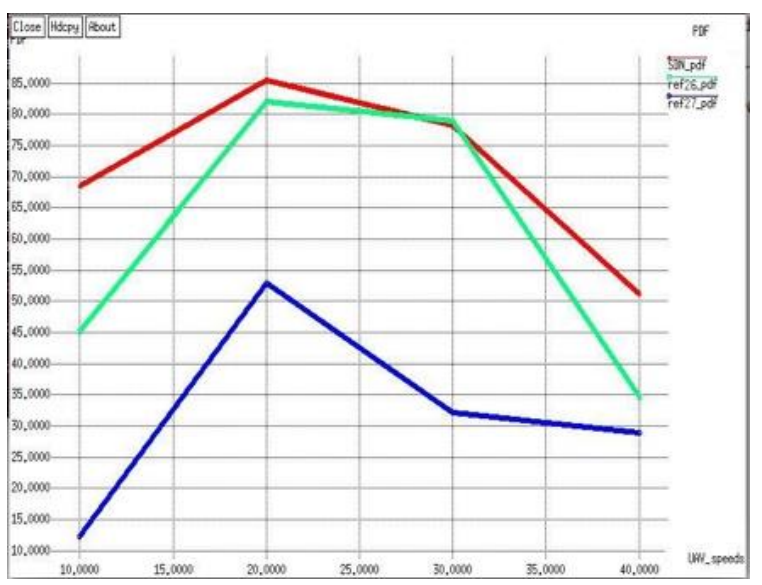

(c)

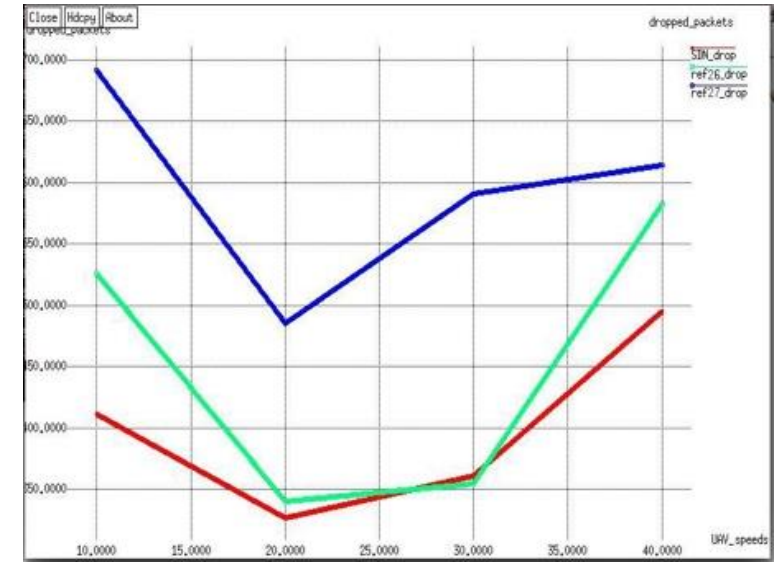

(b)

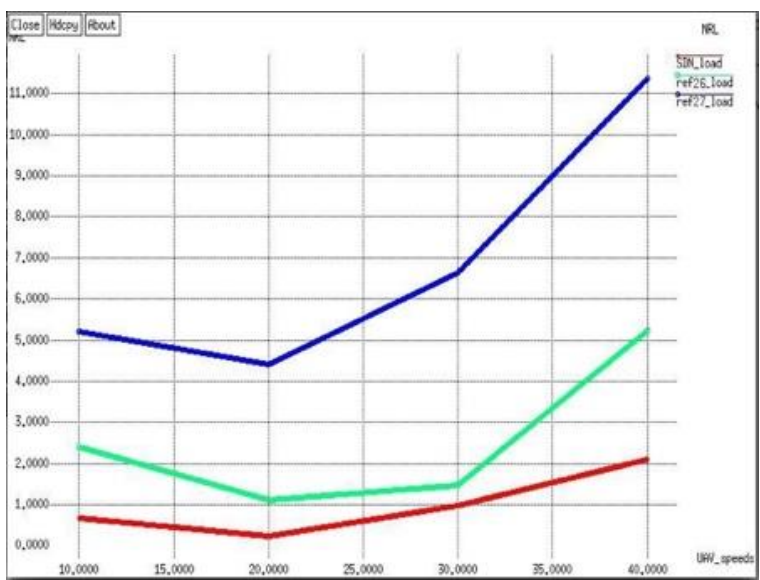

(d)

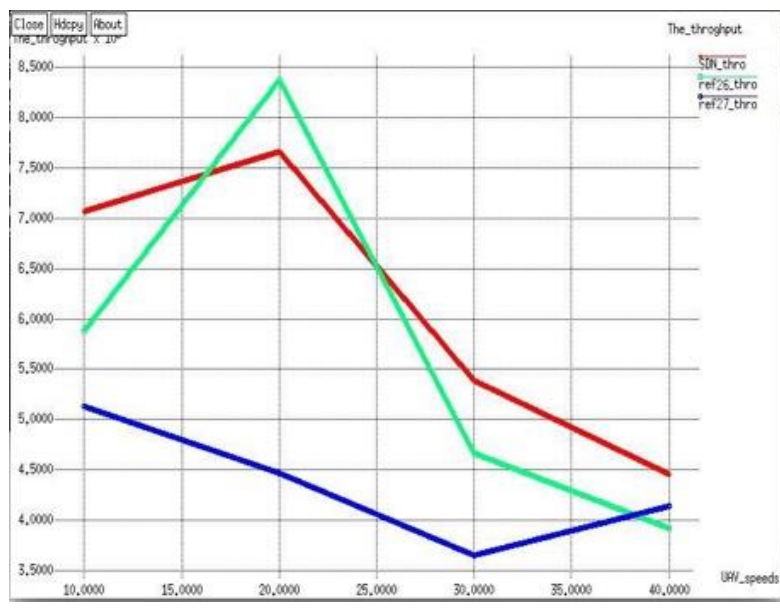

(e)

Figure 5. The performance metrics of ASR-FANET, Ref (25) and Ref (26) for scenario 2; (a) PDF, (b) dropped packet, (c) E2E delay, (d) NRL, (e) throughput

\section{CONCLUSION}

This article introduced the ASR-FANET architecture, a novel routing strategy, which includes an SDN-based topology management and routing of FANET. The primary goal of ASR-FANET is to improve its network efficiency while taking into account all routing constraints, such as rapid changing network topology, high UAV mobility, intermittent links and link quality changes. The performance of ASR-FANET has been evaluated compared to two common adhoc routing protocols (AODV and OLSR). Results of the 
simulation indicate that the proposed framework can reduce the average E2E delay, normalizing routing load and dropped packets, and increase the throughput and packet delivery fraction. In the future work, we plan to extend our framework into optimal FANET topology with SDN strategy in deadline based IoT application.

\section{REFERENCES}

[1] L. Gupta, R. Jain and G. Vaszkun, "Survey of Important Issues in UAV Communication Networks," in IEEE Communications Surveys and Tutorials, vol. 18, no. 2, pp. 1123-1152, Secondquarter 2016, doi: 10.1109/COMST.2015.2495297.

[2] H. Shakhatreh et al., "Unmanned Aerial Vehicles (UAVs): A Survey on Civil Applications and Key Research Challenges," in IEEE Access, vol. 7, pp. 48572-48634, 2019, doi: 10.1109/ACCESS.2019.2909530.

[3] I. Bekmezci, O. K. Sahingoz, and S. Temel, "Flying ad-hoc networks (FANETs): A survey. Ad Hoc Networks," vol. 11, no. 3, pp. 1254-1270, doi: doi.org/10.1016/j.adhoc.2012.12.004.

[4] O. K. Sahingoz, "Networking models in flying ad-hoc networks (FANETs): Concepts and challenges," Journal of Intelligent and Robotic Systems, vol. 74, no. 1-2, pp. 513-527, 2014, doi: 10.1007/s10846-013-9959-7.

[5] M. A. Khan, S. M. Riaz, R. M. Asif, and A. Shah, "Review of communication protocols for FANETs:(Flying adhoc networks)," In International Conference on Engineering and Emerging Technologies, 2015.

[6] M. B. Yassein and N. Alhuda, "Flying ad-hoc networks: Routing protocols, mobility models," International Journal of Advanced Computer Science and Applications (IJACSA), no. 7, no. 6, 2016, doi: 10.14569/IJACSA.2016.070621.

[7] O. K. Sahingoz, "Mobile networking with UAVs: Opportunities and challenges," 2013 International Conference on Unmanned Aircraft Systems (ICUAS), Atlanta, GA, USA, 2013, pp. 933-941, doi: 10.1109/ICUAS.2013.6564779.

[8] M. H. Tareque, M. S. Hossain, and M. Atiquzzaman, "On the routing in Flying Ad Hoc Networks," 2015 Federated Conference on Computer Science and Information Systems (FedCSIS), Lodz, Poland, 2015, pp. 1-9, doi: 10.15439/2015F002.

[9] O. S. Oubbati, M. Atiquzzaman, P. Lorenz, M. H. Tareque and M. S. Hossain, "Routing in Flying Ad Hoc Networks: Survey, Constraints, and Future Challenge Perspectives," in IEEE Access, vol. 7, pp. 81057-81105, 2019, doi: 10.1109/ACCESS.2019.2923840.

[10] Y. Liu, H. N. Dai, Q. Wang, M. K. Shukla, and M. Imran, "Unmanned aerial vehicle for internet of everything: Opportunities and challenges," Computer Communications, vol. 155, pp. 66-83, 2020, doi: 10.1016/j.comcom.2020.03.017.

[11] A. D. Boursianis et al., "Internet of Things (IoT) and Agricultural Unmanned Aerial Vehicles (UAVs) in Smart Farming: A Comprehensive Review," Internet of Things, p. 100187, 2020, doi: 10.1016/j.iot.2020.100187.

[12] M. Mozaffari, W. Saad, M. Bennis, and M. Debbah, "Mobile Unmanned Aerial Vehicles (UAVs) for EnergyEfficient Internet of Things Communications," in IEEE Transactions on Wireless Communications, vol. 16, no. 11, pp. 7574-7589, Nov. 2017, doi: 10.1109/TWC.2017.2751045.

[13] M. F. Khan, K. L. A. Yau, R. M. Noor, and M. A. Imran, "Routing schemes in FANETs: A survey," Sensors, vol. 20 , no. 1 , pp. 38 , doi: 10.3390/s20010038.

[14] M. A. Khan, I. U. Khan, A. Safi, and I. M. Quershi, "Dynamic routing in flying ad-hoc networks using topologybased routing protocols," Drones, vol. 2, no. 3, pp. 27, 2018, doi: 10.3390/drones2030027.

[15] S. Bera, S. Misra and A. V. Vasilakos, "Software-Defined Networking for Internet of Things: A Survey," in IEEE Internet of Things Journal, vol. 4, no. 6, pp. 1994-2008, Dec. 2017, doi: 10.1109/JIOT.2017.2746186.

[16] O. Sami Oubbati, M. Atiquzzaman, T. A. Ahanger, and A. Ibrahim, "Softwarization of UAV Networks: A Survey of Applications and Future Trends," in IEEE Access, vol. 8, pp. 98073-98125, 2020, doi: 10.1109/ACCESS.2020.2994494.

[17] A. Ramaprasath, A. Srinivasan, C. H. Lung, and M. St-Hilaire, "Intelligent wireless ad hoc routing protocol and controller for UAV networks," In Ad Hoc Networks, Springer, Cham, doi: 10.1007/978-3-319-51204-4_8

[18] T. Dapper e Silva, C. F. Emygdio de Melo, P. Cumino, D. Rosário, E. Cerqueira, and E. Pignaton de Freitas, "STFANET: SDN-Based Topology Management for Flying Ad Hoc Network," in IEEE Access, vol. 7, pp. 173499-173514, 2019, doi: 10.1109/ACCESS.2019.2956724.

[19] G. Secinti, P. B. Darian, B. Canberk, and K. R. Chowdhury, "Resilient end-to-end connectivity for software defined unmanned aerial vehicular networks," 2017 IEEE 28th Annual International Symposium on Personal, Indoor, and Mobile Radio Communications (PIMRC), Montreal, QC, Canada, 2017, pp. 1-5, doi: 10.1109/PIMRC.2017.8292772.

[20] H. Iqbal, J. Ma, K. Stranc, K. Palmer, and P. Benbenek, "A software-defined networking architecture for aerial network optimization," 2016 IEEE NetSoft Conference and Workshops (NetSoft), Seoul, Korea (South), 2016, pp. 151-155, doi: 10.1109/NETSOFT.2016.7502462.

[21] Z. Yuan, X. Huang, L. Sun, and J. Jin, "Software defined mobile sensor network for micro UAV swarm," 2016 IEEE International Conference on Control and Robotics Engineering (ICCRE), Singapore, 2016, pp. 1-4, doi: 10.1109/ICCRE.2016.7476140.

[22] W. Qi, Q. Song, X. Kong, and L. Guo, "A traffic-differentiated routing algorithm in Flying Ad Hoc Sensor Networks with SDN cluster controllers," Journal of the Franklin Institute, vol. 356, no. 2, pp. 766-790, 2019, doi: 10.1016/j.jfranklin.2017.11.012

[23] K. Chen, S. Zhao, and N. Lv, "Network monitoring information collection in the SDN-enabled airborne tactical network," International Journal of Aerospace Engineering, 2018, doi: 10.1155/2018/1940842. 
[24] M. Alharthi, A. M. Taha, and H. S. Hassanein, "An Architecture for Software Defined Drone Networks," ICC 2019-2019 IEEE International Conference on Communications (ICC), Shanghai, China, 2019, pp. 1-5, doi: 10.1109/ICC.2019.8761968.

[25] R. Kirichek, A. Vladyko, A. Paramonov, and A. Koucheryavy, "Software-defined architecture for flying ubiquitous sensor networking," 2017 19th International Conference on Advanced Communication Technology (ICACT), PyeongChang, Korea (South), 2017, pp. 158-162, doi: 10.23919/ICACT.2017.7890076.

[26] K. Singh and A. K. Verma, "Experimental analysis of AODV, DSDV and OLSR routing protocol for flying adhoc networks (FANETs)," 2015 IEEE International Conference on Electrical, Computer and Communication Technologies (ICECCT), Coimbatore, India, 2015, pp. 1-4, doi: 10.1109/ICECCT.2015.7226085.

[27] S. Rosati, K. Krużelecki, G. Heitz, D. Floreano, and B. Rimoldi, "Dynamic Routing for Flying Ad Hoc Networks," in IEEE Transactions on Vehicular Technology, vol. 65, no. 3, pp. 1690-1700, March 2016, doi: 10.1109/TVT.2015.2414819.

\section{BIOGRAPHIES OF AUTHORS}
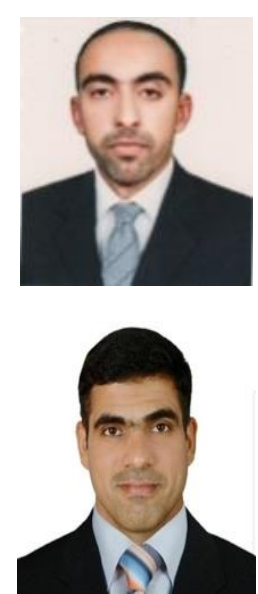

Alaa Taima Albu-salih received the B.Sc. degree in Computer Engineering from University of Al-Qadisiyah, Iraq in 2005, the M. Sc degree in Information Technology from Babylon University, Iraq in 2013 and the Ph.D. degree in Computer Engineering at Ferdowsi university of Mashhad, Mashhad, Iran in 2018. His research interests include wireless sensor networks, mobility models, routing, software-defined networking, internet of things, mathematical modeling, optimization, Unmanned Aerial Vehicle and he have several publications in these fields.

Hayder Ayad Khudhair is completed B.Sc. in Computer Sciences at Babylon University, Babil, Iraq. Receive his M. Sc (master) in Computer Sciences in the year of 2014 from the college of computer sciences at Babylon University as well his Phd. degree from Babylon University, college of information technology in 2019. He is currently working as a lecturer in Al-Najaf Education Directorate. His research in master degree interests Ad-Hoc networks latency time and his research in Phd. degree interests Wireless Sensor Network coverage and connectivity, data mining, machine learning as regression analysis and he have several publications in these fields. 